\title{
A simplified analytical model for post-processing experimental results from tube bulging test: Theory, experimentations, simulations
}

\author{
Nathalie Boudeau ${ }^{a, b, n}$, Pierrick Malécot ${ }^{\text {a,b }}$ \\ a ENSMM, 26 rue Epitaphe, 25030 Besanc-on Cedex, France \\ b FEMTO-ST, Department of Applied Mechanics, 24 rue Epitaphe, 25000 Besanc-on, France
}

A complete analytical model combined with a very simple experimental procedure is proposed. It permits the post-processing of experimental measures to obtain the stress-strain curve for tubes very quickly and well adapted for industrial use. The quality of the results is proved by comparison with experimental measures and finite element results. Anisotropy in tube is revealed by plotting the $(r, a)$ curve where $r$ and a stand for strain and stress path respectively. Two quadratic criteria (Hill 1948 and Hill 1993) are studied and it is found that the Hill 1993 criterion seems the best to represent tube anisotropy for 316L stainless steel tube studied in the present paper.

\section{Introduction}

Tube hydroforming process consists in forming a tube inside a closed and shaped cavity by an internal pressure. For complex shapes and thinning limitation, a combination of an internal pressure and a compression axial force is needed. This technology presents a great industrial interest because it permits to obtain complex hollow shaped parts with a reduced number of welding spots and higher structural quality [1-4]. This process is particularly developed in competitive industries where finite element simulations are intensively used to decrease lead time for design. For that, efficient FE models are needed and it is well known that material data still represents a critical point. Too often, simulations are based on material characteristics obtained from tensile test done on flat sheet specimen. These material data present several limitations: (1) for a same material grade, one cannot compare a flat sheet with a tube; (2) engineer strains are limited to around $20 \%$ due to necking that is very low compared to the deformation possibilities for the loading conditions in the hydroforming process; (3) for advanced steels (like TRiP steels for example), plastic behaviour strongly depends on strain path.

\footnotetext{
* Corresponding author at: ENSMM, 26 rue Epitaphe, 25030 Besançon Cedex, France. Tel.: +33 381666 034; fax: +33 381666700 .

E-mail addresses: Nathalie.Boudeau@ens2m.fr (N. Boudeau), Pierrick.Malecot@ens2m.fr (P. Malécot).
}

By analogy with bulge test for sheets, the tube bulging test is recommended for material characterisation dedicated to tube hydroforming. A tube clamped at its two extremities is put under an internal pressure and freely expands along a called "free zone" (Fig. 1).

To get material data from these tests, it is necessary to develop specific model and no standard is defined at the present time [5]. So several authors have proposed different approaches for the experimental data post-processing that can be classified into three families: (1) approaches based on 'off-line' measurements [6,7], (2) approaches based on 'on-line' measurements [8-11] and (3) approaches based on a mix of 'on-line' and 'off-line' measurements [12,13]. The first and third families are not satisfying because the approaches are very time and material consuming. Some models are based on strong assumptions such as hardening law $[6,12]$, thickness evolution $[9,10]$. Other approaches need FE simulations and iterative methods [6,9].

Moreover, the tube bulging test is quite complex and several sources of uncertainty exist. It is then important to be able to quantify the uncertainty on the resulting material data. For that rapid procedure is needed if global sensitivity method is planned to be conducted.

In the present paper, an evolution of the Velasco and Boudeau model [11] is proposed. In [11] a semi-analytical model was suggested. The resolution method was based on a NewtonRaphson algorithm. Therefore, it is not well adapted for the evaluation of errors on the resulting hardening curve. So a 
complete analytical modelling combined with a very simple experimental procedure is proposed in Section 2 and provide an experimental method adapted to industrial context to get the strain-stress curve. In Section 3 experimental and numerical works are presented. Results and discussion can be found in Section 4.

\section{Theory}

\subsection{Geometrical representation}

The first step for the establishment of the analytical model is a geometrical representation of a bulged tube. From the observation done on FE simulations of the tube bulging test, it permits to postulate that its longitudinal profile can be approached by an arc of circumference. So the parameterisation described in Fig. 2 can be proposed. The definition of the different parameters is given in Table 1.

From Fig. 2, the following geometrical parameters can be evaluated:

$R=\frac{h^{2}+d^{2}}{2 h}$
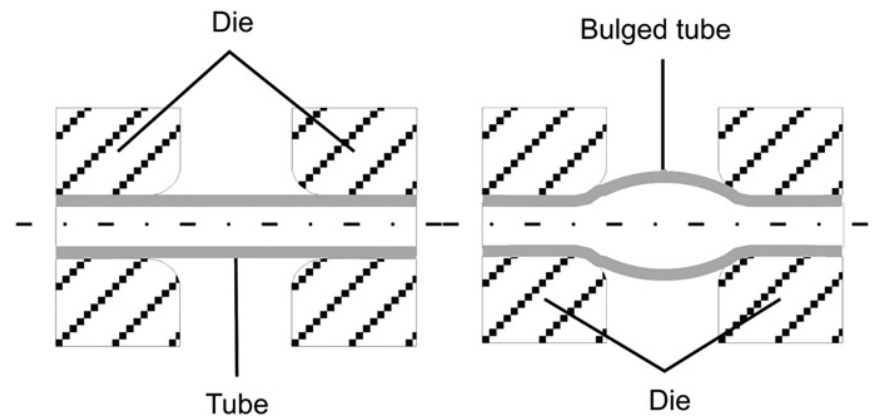

Fig. 1. The tube bulging test: a schematic description.

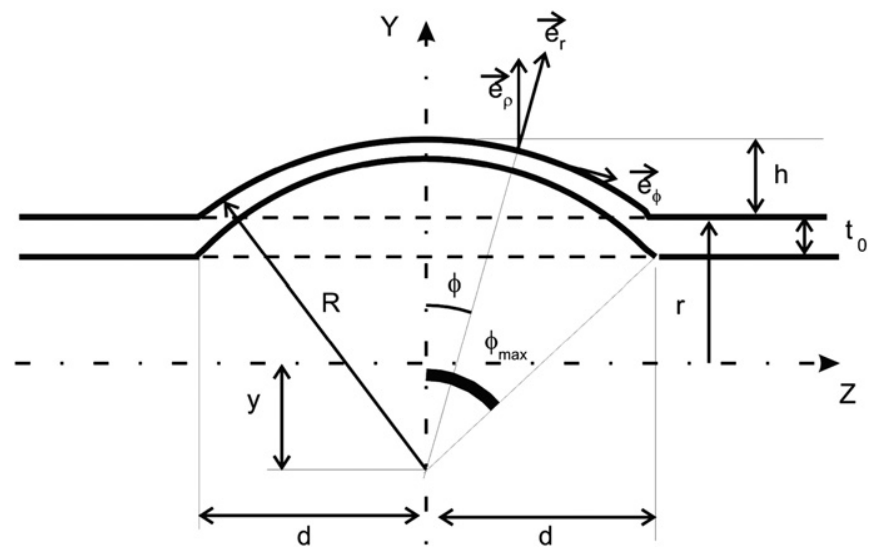

Fig. 2. Parameterisation of the tube bulging test.

Table 1

List of the parameters used for the tube bulging test representation.

\begin{tabular}{ll}
\hline$r$ & Initial tube radius (given) \\
$d$ & Half length of free bulging (given) \\
$t_{0}$ & Initial tube thickness (given) \\
$h$ & Bulging height (measured) \\
$R$ & Radius of the arc of circumference (calculated) \\
$y$ & Position/coordinate of the centre of the arc of circumference (calculated) \\
$\phi_{\max }$ & Angular sector of the arc of circumference (calculated)
\end{tabular}

$y=r+h-R<0$

$\sin \left(\phi_{\max }\right)=\frac{d}{R}$

It is also possible to calculate the coordinates for each point of the tube profile

$Z(\phi)=R \sin \phi$

$Y(\phi)=y+\sqrt{R^{2}-Z(\phi)^{2}}$

\subsection{Strains calculation}

For strains evaluation a local frame $\left(\overrightarrow{e_{\phi}}, \overrightarrow{e_{\theta}}, \overrightarrow{e_{r}}\right)$ is defined as illustrated in Fig. 3. In this set of axis the strain tensor takes the following form:

$\left.\underline{\varepsilon}(M)=\begin{array}{ccc}\varepsilon_{\phi \phi}(\phi) & 0 & 0 \\ 0 & \varepsilon_{\theta \theta}(\phi) & 0 \\ 0 & 0 & \varepsilon_{r r}(\phi)\end{array}\right) \underset{\left(\overrightarrow{e_{\phi}}, \overrightarrow{e_{\theta}}, \overrightarrow{e_{r}}\right)}{\longrightarrow}$

where $\varepsilon_{\phi \phi}$ is the true longitudinal strain in the tube, $\varepsilon_{\theta \theta}$ the true circumferential strain and $\varepsilon_{r r}$ the true radial strain.

They can be calculated as following:

$\varepsilon_{\phi \phi}(\phi)=\ln \left(\frac{R \phi_{\max }}{d}\right)$

$\varepsilon_{\theta \theta}(\phi)=\ln \left(\frac{Y(\phi)}{r}\right)$

then $\varepsilon_{\phi \phi}$ does not depend on $\phi$ and is constant along the tube length.

The last strain tensor component can be evaluated by using the incompressibility condition

$\varepsilon_{r r}(\phi)=-\varepsilon_{\phi \phi}(\phi)-\varepsilon_{\theta \theta}(\phi)$

Finally, from Eq. (9) the current thickness can be calculated

$t(\phi)=t_{0} \exp \left[\varepsilon_{r r}(\phi)\right]$

\subsection{Stress calculation}

In the same set of axis, by considering thin tube, the stress tensor can be expressed as follows:

$$
\left.\underline{\sigma}(M)=\begin{array}{ccc}
\sigma_{\phi \phi}(\phi) & 0 & 0 \\
0 & \sigma_{\theta \theta}(\phi) & 0 \\
0 & 0 & 0
\end{array}\right) \underset{\left(\overrightarrow{e_{\phi}}, \overrightarrow{e_{\theta}}, \overrightarrow{e_{r}}\right)}{\longrightarrow}
$$

For the evaluation of stress components, equilibriums of elementary volumes of tube under pressure are studied. The two infinitesimal parts of tube considered for these evaluations are given in Fig. 4. From these mechanical equilibriums the two following equations are obtained:

$t(\phi) Y(\phi) \cos (\phi) \sigma_{\phi \phi}(\phi)-\frac{1}{2} p[Y(\phi)]^{2}=C$

$\frac{\sigma_{\theta \theta}(\phi)}{Y(\phi)}+\cos (\phi) \frac{\sigma_{\phi \phi}(\phi)}{R}=\frac{p}{t(\phi)}$

where $p$ is the internal pressure inside the tube and $C$ a constant.

To obtain the stress tensor components, it is essential to get a value for the constant $C$. For that, the mechanical equilibrium of a half longitudinal slice of tube with no thickness is considered (Fig. 5). It gives the following equations:

$$
\left\{\begin{array}{l}
\sigma_{\phi \phi}\left(\phi_{\max }\right) t\left(\phi_{\max }\right) \sin \left(\phi_{\max }\right)=p d \\
\sigma_{\phi \phi}(0) t(0)=p h+\sigma_{\phi \phi}\left(\phi_{\max }\right) t\left(\phi_{\max }\right) \cos \left(\phi_{\max }\right)
\end{array}\right.
$$




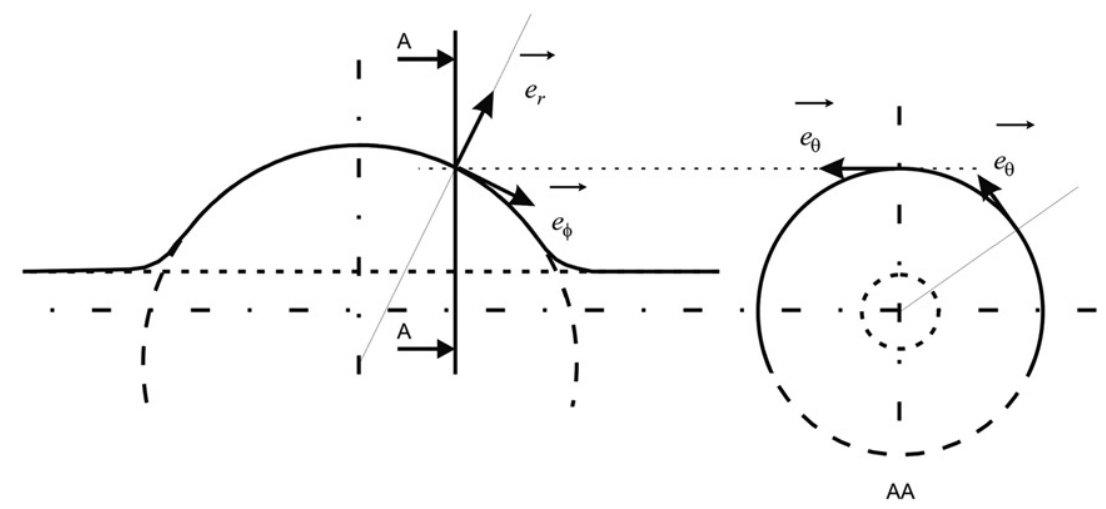

Fig. 3. Definition of the local set of axis $\left(\overrightarrow{e_{\phi}}, \overrightarrow{e_{\theta}}, \overrightarrow{e_{r}}\right)$ (left: tube profile; right: section AA).
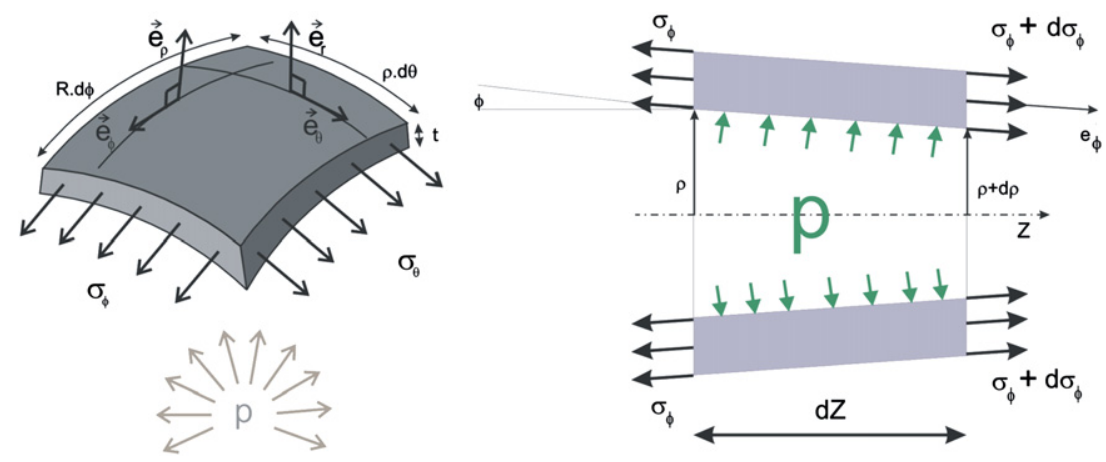

Fig. 4. The two infinitesimal parts of tube considered for stresses evaluation.

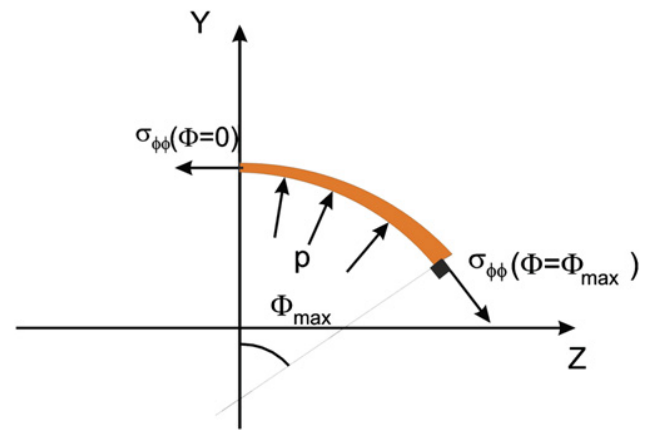

Fig. 5. Equilibrium of a half longitudinal slice of tube in a bulging test.

So the longitudinal stress at the pole is

$\sigma_{\phi \phi}(0)=\frac{p}{t(0)}\left(h+\frac{d}{\tan \left(\phi_{\max }\right)}\right)$

Eq. (15) can lead to numerical problem when $\phi_{\max }$ is close to zero i.e. when the deformed tube curvature is weak. In that case, the hypothesis, already used in [11], is applied; the longitudinal stress at the pole is approached by the longitudinal stress of a tube (radius: $R$; thickness: $e$ ) under pressure closed at its two ends, $\sigma_{z z}=(p \rho / 2 e)$ where $\rho=r+h$ and $e=t(0)$. This corresponds to an approximation for thin tube of relations found in the literature $[14,15]$. The compression force applied to the tube to realize the tightness does not create plastic strain in the free zone of the tube and is then neglected for stress evaluation.

The limit between enough or not enough curvature is given by the angle $\phi_{\max }^{\lim }$ such as

$\tan \left(\phi_{\max }^{\lim }\right)=\frac{2 d}{r-h}$
So, $C$ constant can be calculated as following:

$\left\{\begin{array}{l}C=0, \quad \text { if } \phi<\phi_{\max }^{\lim } \\ C=t(0)(r+h) \sigma_{\phi \phi}(0)-\frac{1}{2} p(r+h)^{2}, \quad \text { if } \phi \geq \phi_{\max }^{\lim }\end{array}\right.$

and the stress tensor components are calculated as following:

$$
\begin{aligned}
\sigma_{\phi \phi}(\phi) & =\frac{C+\frac{1}{2} p[Y(\phi)]^{2}}{t(\phi) Y(\phi) \cos (\phi)} \\
\sigma_{\theta \theta}(\phi) & =Y(\phi)\left[\frac{p}{t(\phi)}-\cos (\phi) \frac{\sigma_{\phi \phi}(\phi)}{R}\right]
\end{aligned}
$$

\subsection{Algorithm for experimental results post-processing}

Eqs. (1)-(19) are used for the calculation of strain and stress tensors components from pressure $p$ and bulge height $h$ measurements. The algorithm presented in Fig. 6 has been carried out with Matlab $^{\odot}$ and its run is very rapid so interesting for an industrial use.

\section{Experiments and simulations}

\subsection{Tube and material}

Experiments are conducted on a tube made of a 316L stainless steel whose dimensions are reported in Table 2. Tubes are obtained by cold drawing.

Data on 316L stainless steel can be found in the literature or on the Web for sheets; they are listed in Tables 3-5. 


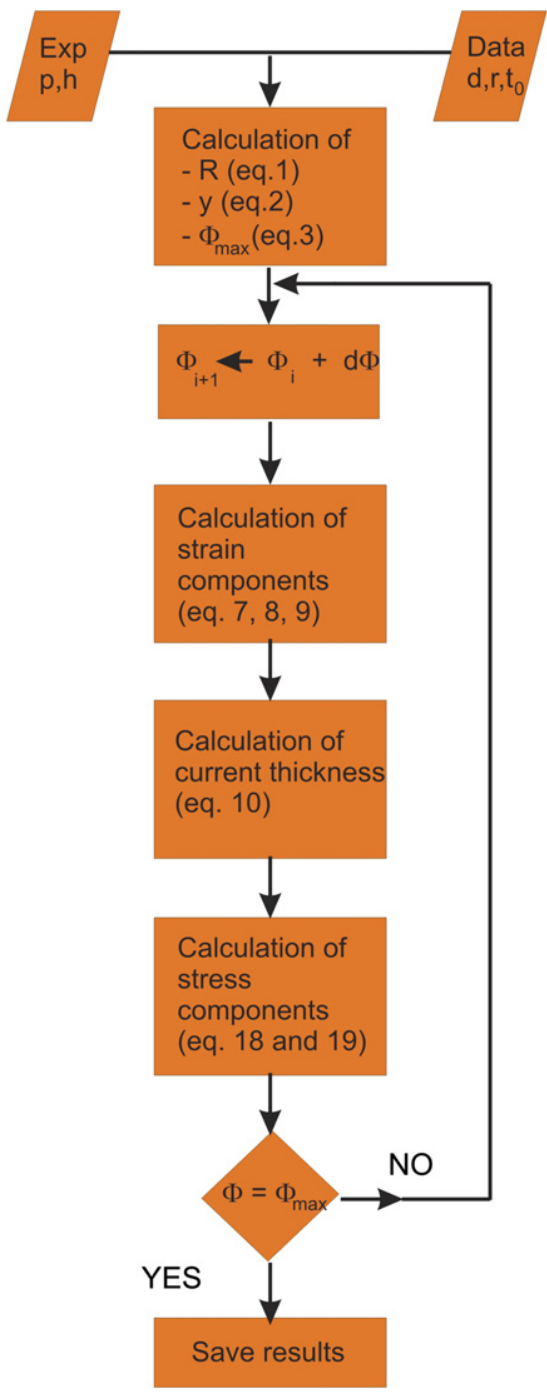

Fig. 6. Algorithm for strains and stresses evaluation in a tube bulging test.

Table 2

Dimensions of tube.

\begin{tabular}{llll}
\hline Length $(\mathrm{mm})$ & External radius $(\mathrm{mm})$ & Thickness $(\mathrm{mm})$ & $d(\mathrm{~mm})$ \\
\hline 250 & 17.5 & 1 & 25 \\
\hline
\end{tabular}

Table 3

Chemical composition.

\begin{tabular}{lllllllll}
\hline From & $\mathrm{C}(\%)$ & $\mathrm{Cr}(\%)$ & $\mathrm{Ni}(\%)$ & $\mathrm{Mn}(\%)$ & $\mathrm{P}(\%)$ & $\mathrm{Si}(\%)$ & $\mathrm{S}(\%)$ & $\mathrm{Mo}(\%)$ \\
\hline$[17]$ & 0.03 & 17 & 12 & 2 & 0.045 & 1 & 0.03 & 2.50 \\
{$[16]$} & 0.03 & 18.5 & 11.2 & 1.5 & & 0.5 & & 2.25 \\
\hline
\end{tabular}

Table 4

Mechanical characteristics.

\begin{tabular}{llllll}
\hline From & Density $\left(\mathrm{kg} / \mathrm{mm}^{3}\right)$ & $R_{m}(\mathrm{MPa})$ & $R_{p 0.2}(\mathrm{MPa})$ & $A(\%)$ & $E(\mathrm{GPa})$ \\
\hline$[17]$ & 8000 & 585 & 380 & 45 & 193 \\
{$[16]$} & & 610 & 320 & 48 & \\
\hline
\end{tabular}

Table 5

Anisotropy data (from [16]).

\begin{tabular}{lllll}
\hline$r_{0}$ & $r_{45}$ & $r_{90}$ & $r_{\text {moy }}$ & $\Delta r$ \\
\hline 0.75 & 1.17 & 0.91 & 0.94 & -0.34 \\
\hline
\end{tabular}

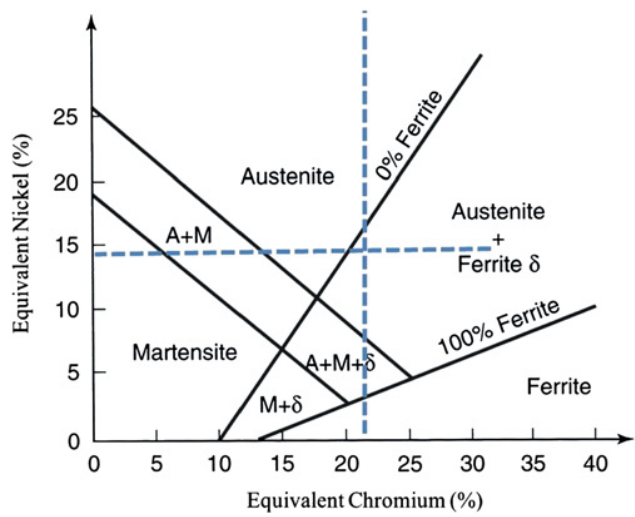

Fig. 7. Schaeffler's diagram (from [18]).

From Schaeffler's diagram (Fig. 7), 316L stainless steel presents an initial austenite-ferrite structure close to the austenitemartensite zone and then can be subject to phase transformation during deformation. The equivalent mass percentage of $\mathrm{Cr}$ and $\mathrm{Ni}$ are calculated with the following relations:

$\mathrm{Cr}_{\text {eq }}=\% \mathrm{Cr}+\% \mathrm{Mo}+1.5(\% \mathrm{Si})+0.5(\% \mathrm{Nb})$

$\mathrm{Ni}_{\text {eq }}=\% \mathrm{Ni}+30(\% \mathrm{C})+0.5(\% \mathrm{Mn})$

In [16], phase transformation is inexistent for tensile tests but has been reported for sequential tensile-shear strain paths.

\subsection{Experimental procedure}

The tool (Fig. 8) is designed in two parts where are machined:

- cylindrical guides for tube positioning,

- conical machining for tube embedding,

- a cavity for tube free bulging, and

- lateral guides for displacement sensor positioning.

Embedding and tightness are insured by a local deformation of the tube in the conical machining with the conical plungers. The upper plunger is drilled to permit fluid feeding and a pressure sensor is integrated to measure the pressure inside the tube.

During the bulge test, internal pressure and pole height of the tube (also named the bulge height) are measured resulting in a pressure-bulge height curve represented in Fig. 9(a). From this curve and the model developed in Section 2 by considering an isotropic material, the true stress-true strain curve of Fig. 9(b) can be obtained. In that case, we have

$\bar{\sigma}^{V M}=\sqrt{\sigma_{\theta \theta}^{2}+\sigma_{\phi \phi}^{2}-\sigma_{\theta \theta} \sigma_{\phi \phi}}$

$\bar{\varepsilon}^{V M}=\frac{2}{\sqrt{3}} \sqrt{\varepsilon_{\theta \theta}^{2}+\varepsilon_{\phi \phi}^{2}+\varepsilon_{\theta \theta} \varepsilon_{\phi \phi}}$

In Fig. 9(b), the resulting hardening curve is compared to the one obtained on 316L sheets with tensile and shear tests [16]. The level of equivalent strain is higher which is in accordance with general observations on bulging test. The level of maximum stress is comparable to the one obtained with shear test and lower than the one obtained with tensile test. 
a

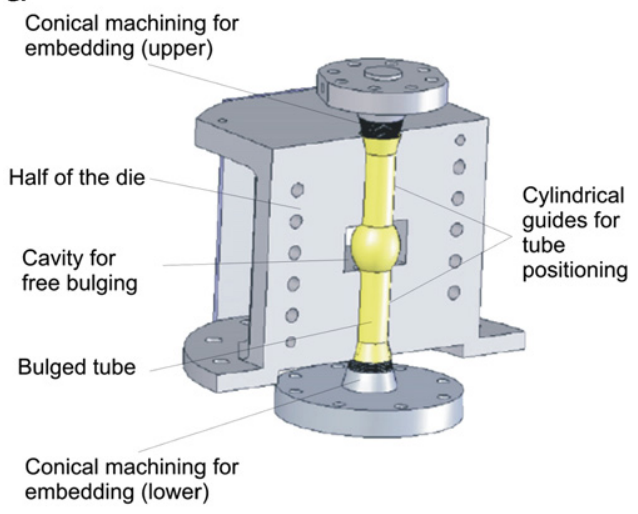

b

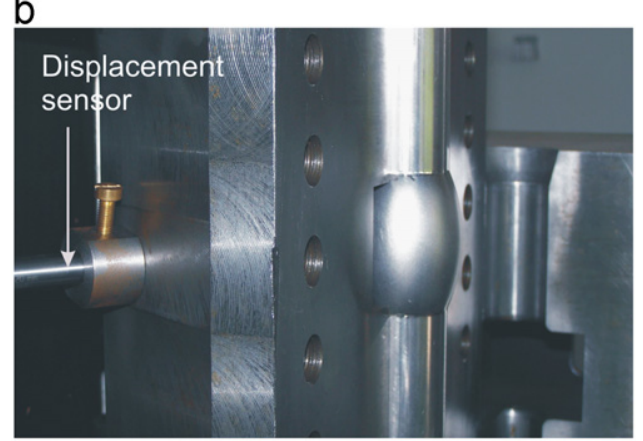

Fig. 8. Tool used for tube bulging test (a) CAD model and (b) photograph of the experimental set-up.

\subsection{Finite element simulations}

Finite element simulations are performed with LS-Dyna ${ }^{\circ}$ program. The full geometry is modelled and meshed with Belytschko-Tsay elements (Fig. 10(a)). Pressure loading is imposed using experimental results. The material behaviour is elastic-plastic and isotropic. For the tube, the given hardening law corresponds to data obtained from the tube bulging experiment (plastic part of the stress-strain curve of Fig. 9(b)). The main information on the FE model are summarised in Table 6. Typical results obtained from these FE simulations are illustrated in Fig. 10(b). The full model has been preferred to avoid numerical instability that can be observed sometimes for the nodes of the symmetry plane.

\section{Results and discussion}

\subsection{Global comparisons}

Global comparisons will concern the pressure-bulge height curve (Fig. 11(a)), the tube profile (Fig. 11(b)) and the thickness repartition along the tube (Fig. 11(c)) at the end of the test. Experimental measures and numerical results are crossexamined.

Fig. 11(a) compares the experimental pressure-bulge height curve with those obtained by FE simulations. It shows that the pressure level is well respected, that is normal as the FE simulations are conducted by pressure loading. The level of bulge heights obtained with the experiments and the implicit calculation are in good agreement. The explicit simulation conducts to a higher bulge height that is not realistic. For explicit simulations predefined numerical parameters have been used and the test duration has been arbitrarily chosen to $0.001 \mathrm{~s}$ from authors'
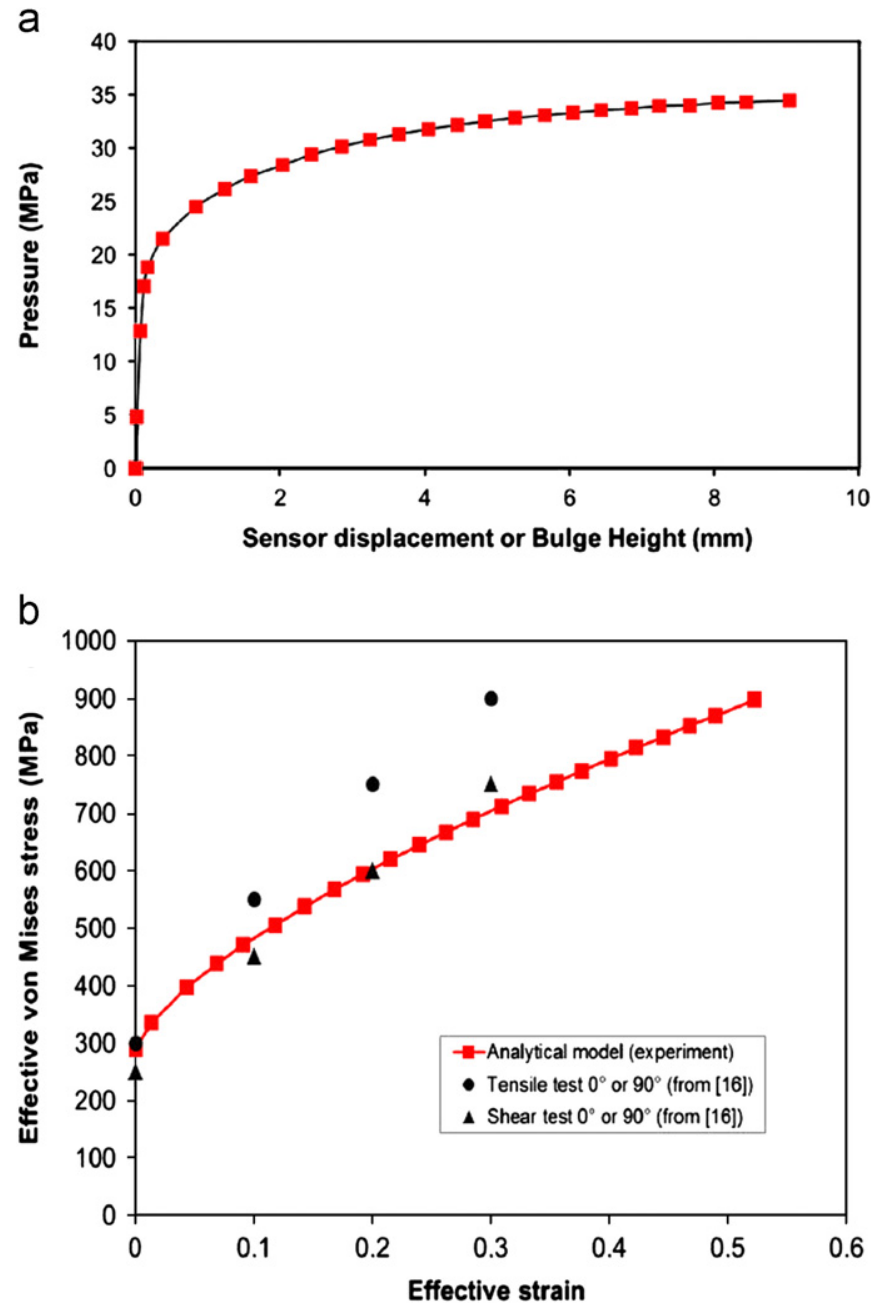

Fig. 9. (a) the pressure-bulge height curve obtained by measurement and (b) the true stress-true strain curve obtained with the model from experiments compared with data obtained on sheet (from [16]).
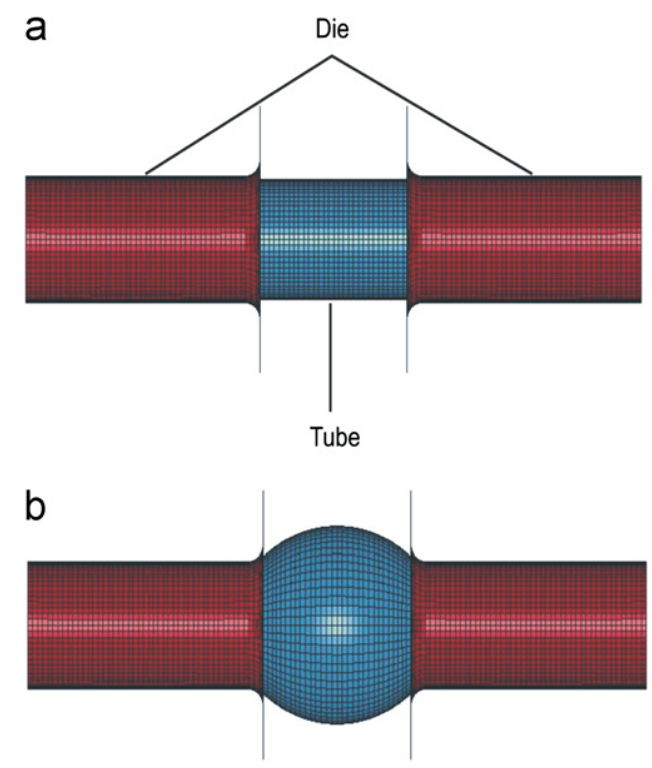

Fig. 10. FE model (a) and typical FE results (b). 
Table 6

Summarise of the FE simulations.

\begin{tabular}{ll}
\hline Tube & Type: shell element \\
& Formulation: Belytschko-Tsay \\
& Average size: $1.7 \mathrm{~mm}$ \\
& Thickness: $1 \mathrm{~mm}$ \\
& Mesh: 8582 nodes and 8404 elements \\
& Material: *MAT_PIECEWISE_LINEAR_PLASTICITY \\
& Type: shell element \\
Die & Average size: $1.7 \mathrm{~mm}$ \\
& Mesh: 7750 nodes and 7688 elements \\
& Material: *MAT_RIGID \\
Contact & 'CONTACT_AUTOMATIC_ONE_WAY_SURFACE_TO_SURFACE \\
algorithm & Pressure law \\
Loading & Implicit and explicit \\
Integration & \\
scheme &
\end{tabular}

experience in explicit FE simulations in process simulations. These choices permit to converge to an acceptable solution for process validation for which implicit codes cannot. But that is not suitable for precise comparisons especially for material data characterisation. For instance, in [6,9] implicit FE simulations are also preferred for identification method or numerical validations.

Fig. 11(b) compares the tube profile at the end of the test measured on the specimen and obtained by FE simulations. Again, the analytical model and the implicit FE simulation present good correlation and the explicit FE calculation gives unrealistic results. The difference between the analytic model and implicit FE simulation are more important near the die radius due to a geometrical simplification for the analytical developments. The same conclusions can be done for Fig. 11(c) where thickness distributions along the bulged tube are plotted.

\subsection{Comparison of strain and stress tensors components}

In order to validate the analytical model proposed in Section 2, it is essential to compare the different models more precisely. In particular, it can be interesting to explore the fields of the strain and stress over the tube at the end of the test.

Fig. 12 presents the repartition of longitudinal and circumferential strain along the bulged tube at the end of the test. The evolution is generally well respected but the level of the circumferential strain is under estimated. Anyway, at the pole position the level of strain is in relatively good correlation with the FE results obtained with an implicit integration scheme. The existing differences are of the same magnitude as those conventionally observed on more common tests as the tensile test for instance. In the analytical model, the longitudinal strain is being kept constant in the whole tube which is not the case in the FE simulations. But the difference with the implicit calculation is not so important, and the value obtained at the maximum of bulge height presents a very good agreement.

Fig. 13 compares the stress tensor components at the end of the bulge test obtained with the three approaches. Again an important difference between the explicit FE simulation and the two other results can be noticed. The results obtained with the analytical model and the implicit FE simulation are very similar. At the pole, the results are quite identical. The divergence between the two approaches is important near the die radius where contact exists. It is difficult to accord more credit to one or another; for the analytical model, die radius and contact notion in this area are ignored; for the FE simulation, contact algorithm has been activated. Contact is certainly the most difficult phenomenon to model in metal forming simulations. Contact energy calculation is based on interface forces, interpenetrations that
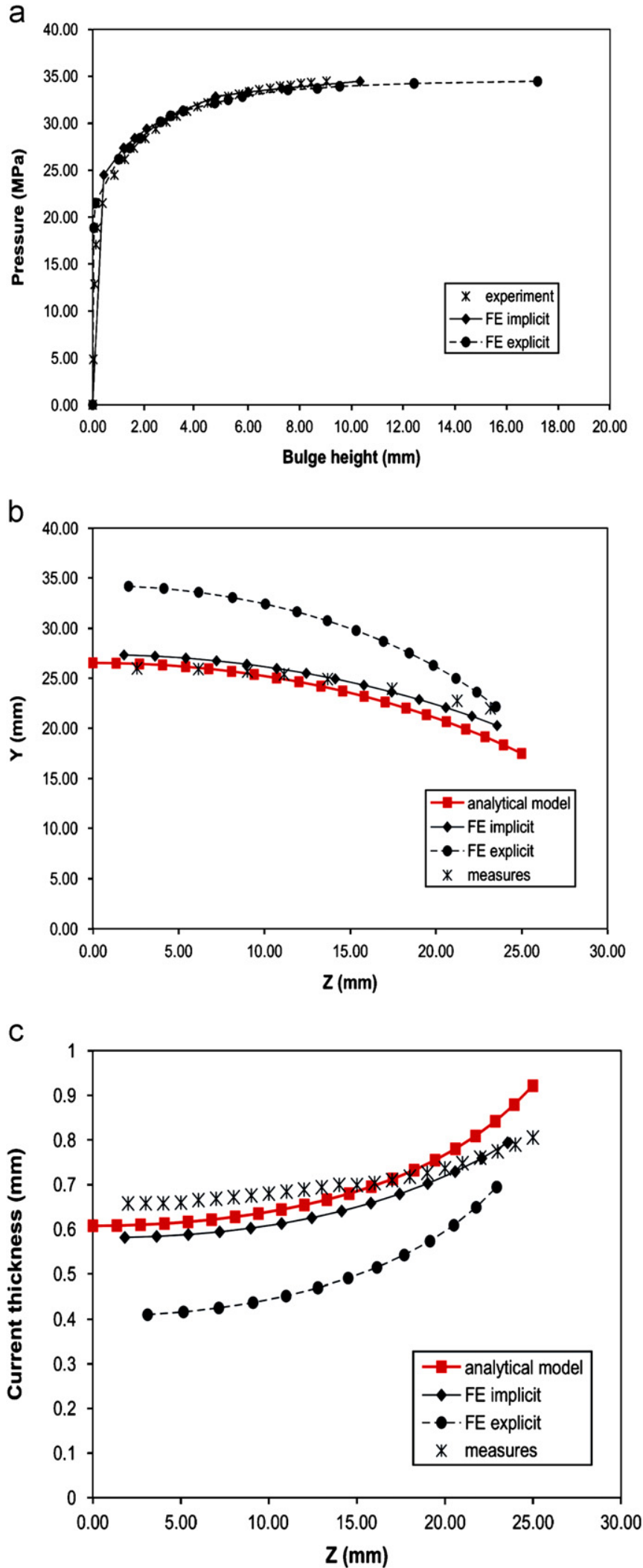

Fig. 11. (a) Pressure-bulge height curve, (b) tube bulge profile and (c) thickness repartition comparisons.

are directly linked to time step and mesh size that are purely numerical entities and do not exist in reality. Friction is modelled with Coulomb's law which is an empirical and macroscopic law 


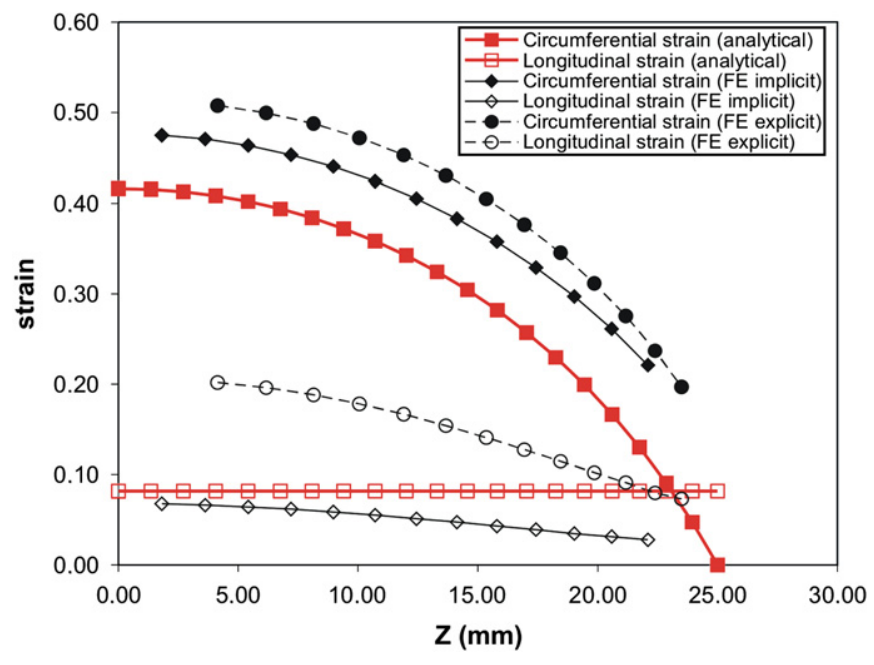

Fig. 12. Longitudinal and circumferential strains along the bulged tube at the end of test.

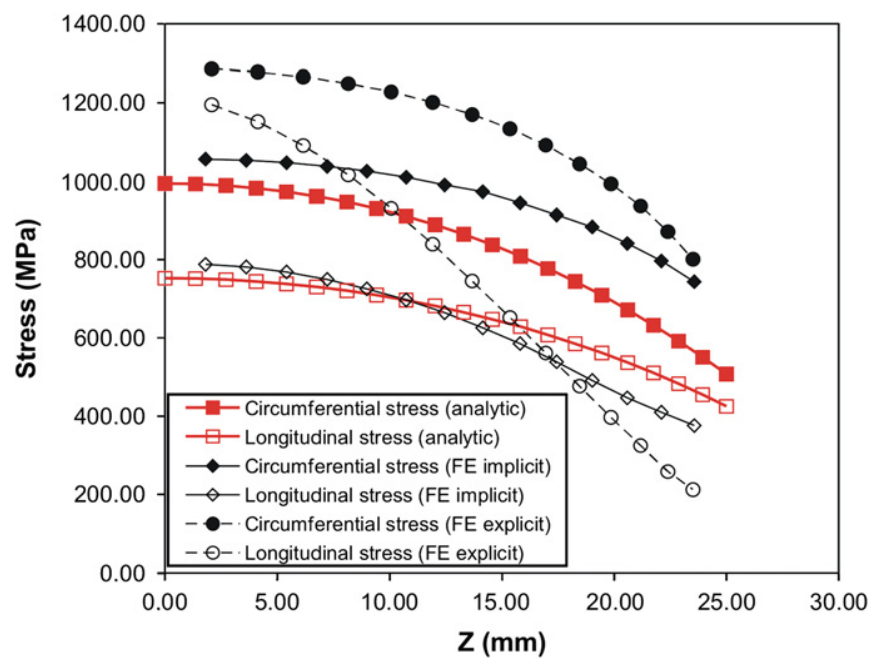

Fig. 13. Longitudinal and circumferential stresses along the bulged tube at the end of test.

that cannot take into account complex physical or chemical phenomena.

\subsection{Strain and stress path}

To go further, investigations on strain and stress path are done. Comparisons will be done only with implicit FE simulations in this section. Strain paths and stress paths at the pole, the die radius and between these two positions are illustrated in Figs. 14 and 15 respectively.

The first observation on strain path (Fig. 14) shows a nonlinearity and tube bulging test appears as a non-proportional test. The strain path is quite well reproduced except at the die radius. The differences of curvature are due to the divergences observed on the strain level in Section 4.2. They can be explained by the fact that the theory ignores the existence of a die radius for the analytical developments. In addition the theory is based on an idealized geometric representation of reality while during testing the actual geometry integrates all physical phenomena such as contact and friction for example.

Curiously, stress paths (Fig. 15) are in better agreement except near the contact zone. Near the die radius (Fig. 15(c)) numerical stress path is very perturbed; this is due to the contact algorithm.
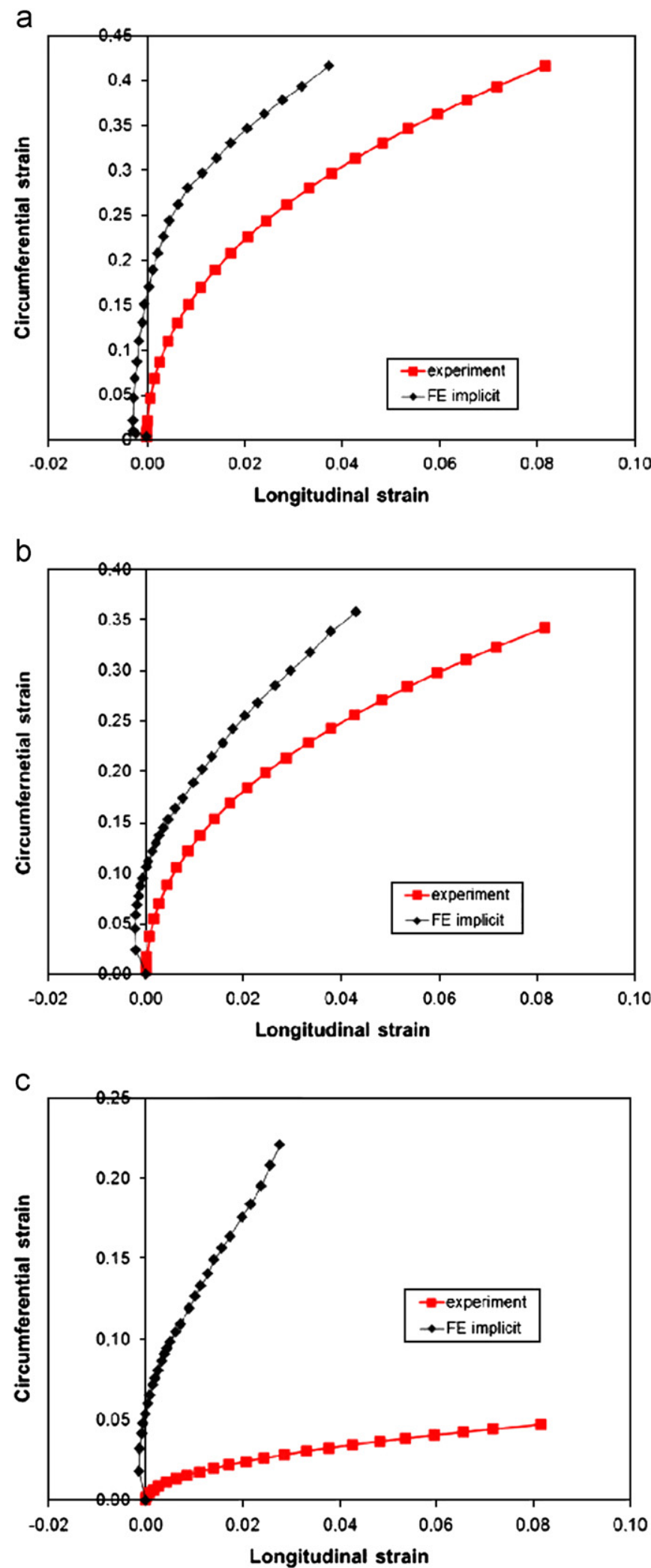

Fig. 14. Strain path (a) at the pole (b) between the pole and the die radius (c) near the die radius.

\subsection{Anisotropy of tube}

Tubes tested in this paper are obtained by drawing. So, they present an initial anisotropy due to their manufacturing process. In order to reveal their anisotropy, the authors propose to plot 

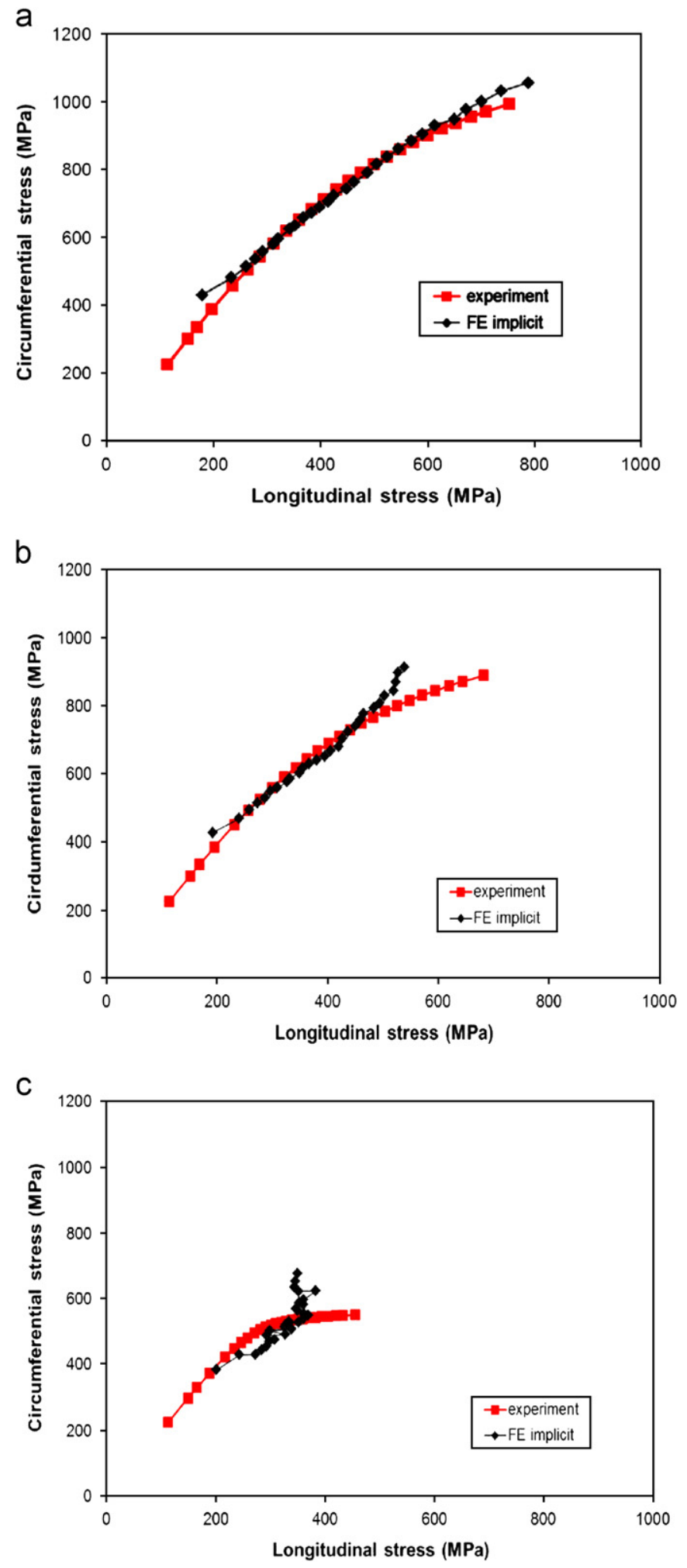

Fig. 15. Stress path (a) at the pole (b) between the pole and the die radius (c) near the die radius.

strain path $\rho=d \varepsilon_{\phi \phi} / d \varepsilon_{\theta \theta}$ vs. stress path $\alpha=\sigma_{\phi \phi} / \sigma_{\theta \theta}$. In previous works on the prediction of necking [19], it was usual to write the relation between stress and strain paths using the plasticity criterion [20]. The resulting expression obtained for the von Mises criterion is shown in Appendix B. The $\left(\rho_{\text {exp }}, \alpha_{\exp }\right)$ and $\left(\rho^{V M}, \alpha^{V M}\right)$ curves are compared in Fig. 16. The method to calculate the $\left(\rho_{\text {exp }}, \alpha_{\exp }\right)$ couple is described in Appendix A. It is evident that the

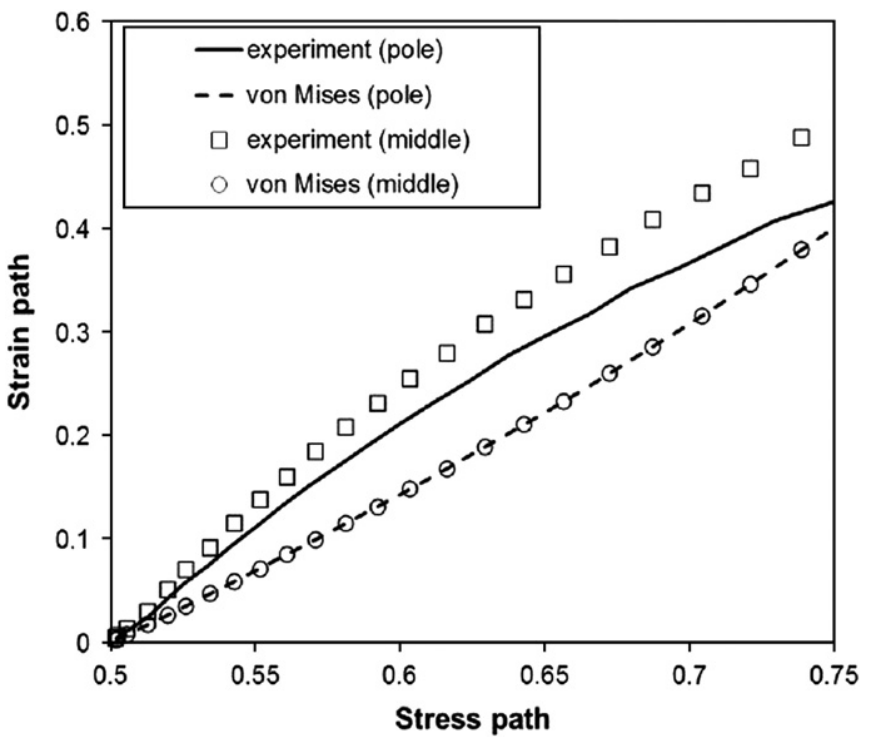

Fig. 16. Revelation of anisotropy of tube by plotting the $(\rho, \alpha)$ curve. Von Mises' results correspond to calculated strain path vs. experimental stress path with the method explained in Appendices A and B.

von Mises' assumption is not suitable for the tested material shaped as tube.

So other plasticity criteria are investigated: Hill 1948 and Hill 1993. Hill 1948 has been chosen because it is classically used for metal forming modelling and is widely implemented in commercial codes. It provides an approximate description of the real yield locus [21]. Hill 1993 criterion is an improvement of plastic behaviour of textured sheet metals submitted to complex load applied along the planar orthotropic axes. It allows describing both the "first order anomalous behaviour" and the "second order anomalous behaviour". It is particularly suitable for biaxial loading conditions but limited to the case where directions of the principal stresses are coincident with the orthotropic axes [21]. That is the case in the tube bulging test.

The following results named "experiment" have been obtained by processing experimental measures.

\subsubsection{Hill 1948 criterion}

From [22], Hill 1948's criterion applied to tubes is

$\bar{\sigma}=\sqrt{\sigma_{\phi \phi}^{2}-\left(1+\frac{1}{S_{\theta}^{2}}-\frac{1}{S_{r}^{2}}\right) \sigma_{\theta \theta} \sigma_{\phi \phi}+\frac{1}{S_{\theta}^{2}} \sigma_{\theta \theta}^{2}+\frac{1}{S_{\phi \theta}^{2}} \sigma_{\phi \theta}^{2}}$

with $S_{\theta}=\left(\sigma_{\theta}^{0} / \sigma_{\phi}^{0}\right), \quad S_{r}=\left(\sigma_{r}^{0} / \sigma_{\phi}^{0}\right), \quad S_{\phi \theta}=\left(\sigma_{\phi \theta}^{0} / \sigma_{\phi}^{0}\right)$.

In our case, the stress tensor has the expression of Eq. (11). Then the criterion takes the simplified expression

$\bar{\sigma}=\sqrt{\sigma_{\phi \phi}^{2}-\left(1+\frac{1}{S_{\theta}^{2}}-\frac{1}{S_{r}^{2}}\right) \sigma_{\theta \theta} \sigma_{\phi \phi}+\frac{1}{S_{\theta}^{2}} \sigma_{\theta \theta}^{2}}$

Hill 1948's criterion is written as following:

$\bar{\sigma}=\sqrt{\sigma_{\phi \phi}^{2}-c \sigma_{\theta \theta} \sigma_{\phi \phi}+a \sigma_{\theta \theta}^{2}}$

where $a=\left(1 / S_{\theta}\right)^{2}, b=\left(1 / S_{r}\right)^{2}$, and $c=1+a-b$.

The plastic flow relation is

$d \varepsilon_{i j}=d \lambda \frac{\partial \bar{\sigma}}{\partial \varepsilon_{i j}}$ 
which gives the relations below

$\left\{\begin{array}{l}d \varepsilon_{\phi \phi}=\frac{d \lambda}{2 \bar{\sigma}}\left[2 \sigma_{\phi \phi}-c \sigma_{\theta \theta}\right] \\ d \varepsilon_{\theta \theta}=\frac{d \lambda}{2 \bar{\sigma}}\left[2 a \sigma_{\theta \theta}-c \sigma_{\phi \phi}\right]\end{array}\right.$ $\alpha$ is

So the relation between the strain path $\rho$ and the stress path

$\rho=\frac{2 \alpha-c}{2 a-c \alpha}$

Anisotropy parameters $a$ and $c$ are searched such as, for the set of data $\left(\rho_{i}, \alpha_{i}\right)$, we must satisfy the following equation:

$2 \rho_{i} a+\left(1-\rho_{i} \alpha_{i}\right) c=2 \alpha_{i}$

\subsubsection{Hill 1993 criterion}

Hill 1993's criterion is expressed (from [21])

$\left(\frac{\sigma_{1}}{\sigma_{0}}\right)^{2}-c\left(\frac{\sigma_{1}}{\sigma_{0}}\right)\left(\frac{\sigma_{2}}{\sigma_{90}}\right)+\left[(p+q)-p \frac{\sigma_{1}}{\sigma_{b}}-q \frac{\sigma_{2}}{\sigma_{b}}\right] \frac{\sigma_{1}}{\sigma_{0}} \frac{\sigma_{2}}{\sigma_{90}}=1$

$\sigma_{1}$ and $\sigma_{2}$ represent the principal stresses. $\sigma_{0}$ and $\sigma_{90}$ represent the elastic yield stresses in a tensile test in $0^{\circ}$ and $90^{\circ}$ directions relatively to the rolling direction, respectively; $\sigma_{b}$ represent the yield stress in a biaxial tensile test.

Criteria are generally built such as $\bar{\sigma}=\sigma_{0}$ in a tensile test in direction $0^{\circ}$. So the criteria can be rewritten as following:

$\bar{\sigma}^{2}=\sigma_{1}^{2}-c A \sigma_{1} \sigma_{2}+\left[(p+q)-B \sigma_{1}-C \sigma_{2}\right] A \sigma_{1} \sigma_{2}$

with $A=\left(\sigma_{0} / \sigma_{90}\right), B=\left(p / \sigma_{b}\right)$, and $C=\left(q / \sigma_{b}\right)$.

In spherical coordinates in reference to the parameterisation of the tube bulging test proposed in Section 2 the Hill 1993 criterion takes the form

$\bar{\sigma}^{2}=\sigma_{\theta}^{2}-c A \sigma_{\theta} \sigma_{\phi}+\left[(p+q)-B \sigma_{\theta}-C \sigma_{\phi}\right] A \sigma_{\theta} \sigma_{\phi}$

The criterion is rewritten as following:

$\bar{\sigma}^{2}=\sigma_{\theta}^{2}+U \sigma_{\theta} \sigma_{\phi}-V \sigma_{\theta}^{2} \sigma_{\phi}-W \sigma_{\phi}^{2} \sigma_{\theta}$

with $U=(p+q-c) A, V=A B$ and $W=A C$.

The plastic flow relation $d \varepsilon_{i j}=d \lambda\left(\partial \bar{\sigma} / \partial \varepsilon_{i j}\right)$ gives the relations below

$\left\{\begin{array}{l}d \varepsilon_{\phi \phi}=\frac{d \lambda}{2 \bar{\sigma}}\left[2 \sigma_{\theta}+U \sigma_{\phi}-2 V \sigma_{\theta} \sigma_{\phi}-W \sigma_{\phi}^{2}\right] \\ d \varepsilon_{\theta \theta}=\frac{d \lambda}{2 \bar{\sigma}}\left[U \sigma_{\theta}-V \sigma_{\theta}^{2}-2 W \sigma_{\theta} \sigma_{\phi}\right]\end{array}\right.$

from which is obtained the relation between $\rho$ and $\alpha$

$\rho=\frac{U-V \sigma_{\theta}-2 W \sigma_{\phi}}{2+U \alpha-2 V \sigma_{\phi}-W \sigma_{\phi} \alpha}$

The anisotropy parameters $U, V$ and $W$ are searched such as, for the set of data $\left(\rho_{i}, \alpha_{i}\right)$, the following equation is verified:

$\left(1-\rho_{i} \alpha_{i}\right) U+\left(2 \rho_{i} \sigma_{\phi}-\sigma_{\theta}\right) V+\left(\alpha_{i} \rho_{i}-2\right) \sigma_{\phi} W=2 \rho_{i}$

\subsubsection{Resolution}

Then the problem to be solved can be expressed

$M_{i j} x_{j}=v_{i}$ with $i \in[1, N]$ and $j \in[1, k]$ where $N$ corresponds to the number of couples $\left(\rho_{i}, \alpha_{i}\right)$ and $k$ the number of parameters defining the plastic criterion.

It takes the matrix form below

$[M] x=v$

and the resolution of the matrix problem is

$x=\left([M]^{T}[M]\right)^{-1}[M]^{T} v$

\subsubsection{Results and discussion}

The anisotropy parameters have been identified by the method presented in previous section. Their values are reported in Table 7 and the resulting $(\rho, \alpha)$ curves are plotted in Fig. 17. The Hill 1993 plastic criterion fits very well the experimental results. From these observations it can be conclude that anisotropy in tube is better represented by Hill 1993's criterion. The resulting hardening curves are compared in Fig. 18 where Hill 1993's criterion leads to higher level of equivalent stress and lower equivalent strain. The maximal strain level reaches quite 0.5 which is higher to 0.3 found on tensile test on sheets in [16]. This is in agreement with classical observations when uniaxial and biaxial tests are compared (see results in [23] for instance). Nevertheless data obtained in Table 7 for Hill 1993 plastic criterion are valid only for the strain path corresponding to the tube bulging test. To obtain intrinsic material characteristics other tests would be needed.

\section{Conclusions}

A complete analytical model combined with a very simple experimental procedure has been proposed. It permits the postprocessing of experimental measures to get the stress-strain

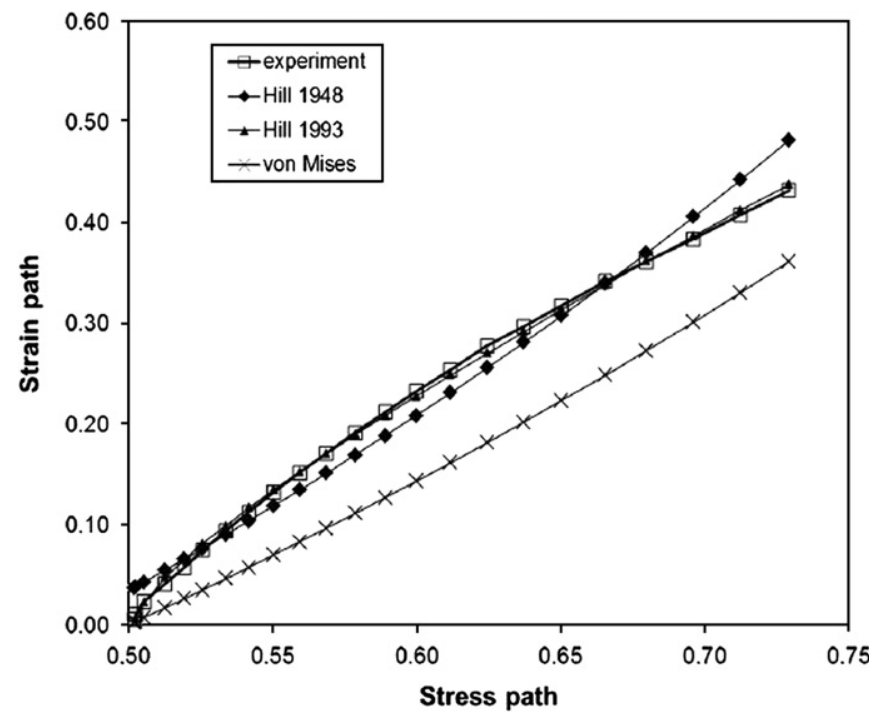

Fig. 17. $(\rho, \alpha)$ curves obtained at the pole for different plastic criteria compared to experimental result. $\alpha$ corresponds to the experimental stress path. Strain path $\rho$ is calculated with relations (28) and (35) for Hill 1948 and Hill 1993 plastic criteria.

Table 7

Identified parameters for Hill 1948 and Hill 1993’s criteria.

\begin{tabular}{|c|c|c|c|c|}
\hline HILL 1948 & $\begin{array}{l}a \\
0.8699\end{array}$ & $\begin{array}{l}c \\
0.9574\end{array}$ & $\begin{array}{l}b \\
0.9125\end{array}$ & $\bar{\sigma}=\sqrt{\sigma_{\phi \phi}^{2}-0.9574 . \sigma_{\theta \theta} . \sigma_{\phi \phi}+0.8699 . \sigma_{\theta \theta}^{2}}$ \\
\hline HILL 1993 & $\begin{array}{l}U \\
-0.1665\end{array}$ & $\begin{array}{l}V \\
4.3 \times 10^{-4}\end{array}$ & $\begin{array}{l}W \\
-9.5587 \times 10^{-4}\end{array}$ & $\bar{\sigma}=\sqrt{\sigma_{\theta}^{2}-0.1665 . \sigma_{\theta} \cdot \sigma_{\phi}-4.3 \times 10^{-4} . \sigma_{\theta}^{2} \cdot \sigma_{\phi}+9.5587 \times 10^{-4} . \sigma_{\phi}^{2} . \sigma_{\theta}}$ \\
\hline
\end{tabular}




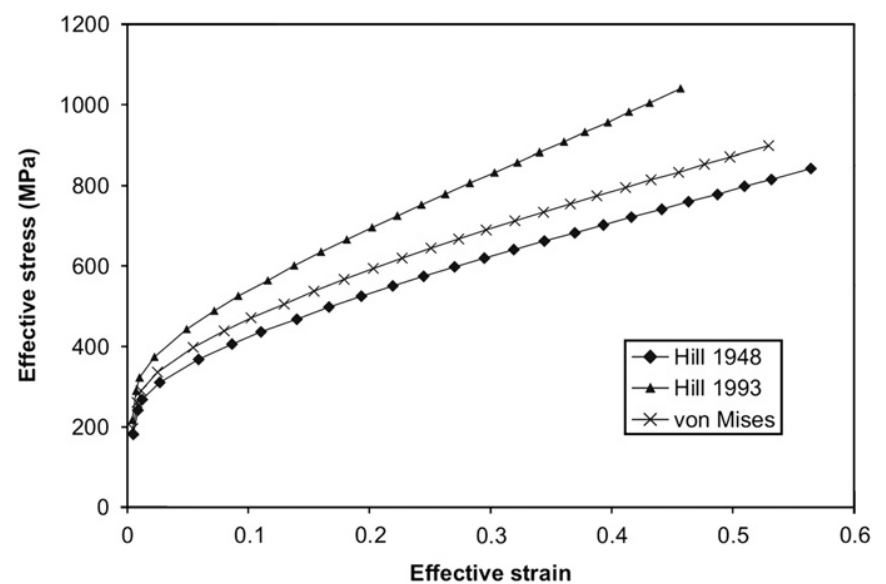

Fig. 18. Comparison of the resulting hardening curves.

curve for tube materials very quickly and well adapted for industrial use.

The quality of the results has been proved by comparing with experimental measures (tube profile, thickness repartition over the bulged tube) or finite element results for strain and stress fields in the tube. Except in the area near the die radius, results obtained with the analytical model, by measures on experimental specimens and by numerical implicit simulations are in good correlation.

This full analytical model will be suitable for studying uncertainties propagation. Thus it will be possible to evaluate uncertainties on the hardening curve due to the ones of the experimental procedure more efficiently than in [24]. In addition it will be adapted for performing global sensitivity analysis (Sobol's type for example) in order to determine advices for experimental works.

Moreover anisotropy in tube has been revealed by plotting the $(\rho, \alpha)$ curve where $\rho$ and $\alpha$ stand for strain and stress path respectively. Two quadratic criteria (Hill 1948 and Hill 1993) have been studied and it is the Hill 1993 plastic criterion that fits better the $(\rho, \alpha)$ curve obtained during experiments. But other tests would be necessary to get intrinsic anisotropic parameters.

\section{Acknowledgement}

The authors thank their colleagues Dr. Sébastien Thibaud for his expertise in FE simulations and Dr. Gérard Michel for his assistance during experiments.

\section{Appendix A. $\left(\rho_{\text {exp }}, \alpha_{\text {exp }}\right)$ plot}

Time of experiment is discretized into $N$ intervals. Current time $t_{i}$ is defined by

$t_{i}=\frac{T}{N} i$

where $T$ corresponds to the total duration of the experiment and $i \in[1 N]$.

The experimental strain and stress paths are evaluated as following:

$\rho_{\exp }\left(t_{i}\right)=\frac{d \varepsilon_{\phi \phi}}{d \varepsilon_{\theta \theta}}\left(t_{i}\right) \approx \frac{\Delta \varepsilon_{\phi \phi}\left(t_{i}\right)}{\Delta \varepsilon_{\theta \theta}\left(t_{i}\right)}=\frac{\varepsilon_{\phi \phi}\left(t_{i+1}\right)-\varepsilon_{\phi \phi}\left(t_{i}\right)}{\varepsilon_{\theta \theta}\left(t_{i+1}\right)-\varepsilon_{\theta \theta}\left(t_{i}\right)}$

$$
\alpha_{\exp }\left(t_{i}\right)=\frac{\sigma_{\phi \phi}\left(t_{i}\right)}{\sigma_{\theta \theta}\left(t_{i}\right)}
$$

Appendix B. $\left(\rho^{V M}, \alpha^{V M}\right)$ plot

With von Mises assumption, the equivalent strain and stress are defined by

$$
\begin{aligned}
& d \bar{\varepsilon}^{V M}=\frac{2}{\sqrt{3}} \cdot \sqrt{d \varepsilon_{\theta \theta}^{2}+d \varepsilon_{\phi \phi}^{2}+d \varepsilon_{\theta \theta} d \varepsilon_{\phi \phi}} \\
& \bar{\sigma}^{V M}=\sqrt{\sigma_{\theta \theta}^{2}+\sigma_{\phi \phi}^{2}-\sigma_{\theta \theta} \sigma_{\phi \phi}}
\end{aligned}
$$

There are linked to the components of the strain and stress tensor by the relation

$\bar{\sigma}^{V M} d \bar{\varepsilon}^{V M}=\underline{\sigma}: d \underline{\varepsilon}$

It gives

$\rho^{V M}=\frac{2 \alpha^{2}-5 \alpha+2}{4 \alpha-\alpha^{2}-4} \quad$ where $\alpha=\alpha_{\exp }$

$\left(\rho^{V M}, \alpha^{V M}=\alpha_{\exp }\right)$ curve is obtained with relation.

\section{References}

[1] Ahmetoglu M, Altan T. Tube hydroforming: state-of-the-art and future trends. J Mater Process Technol 2000;98:25-33.

[2] Koç M, Altan T. An overall review of the tube hydroforming (THF) technology. J Mater Process Technol 2001;108:384-93.

[3] Mac Donald BJ, Hashmi MSJ. Near-net-shape manufacture of engineering components using bulge-forming processes: a review. J Mater Process Technol 2002;120:341-7.

[4] Lang LH, Wang ZR, Kang DC, Yuan SJ, Zhang SH, Danckert J, et al. Hydroforming highlights: sheet hydroforming and tube hydroforming. J Mater Process Technol 2004;151:165-77.

[5] Koc M, Billur E, Cora ON. An experimental study on the comparative assessment of hydraulic bulge test analysis methods. Mater Des 2011;32: 272-81.

[6] Sokolowski T, Gerke K, Ahmetoglu M, Altan T. Evaluation of tube formability and material characteristics: hydraulic bulge testing of tubes. J Mater Process Technol 2000;98:34-40.

[7] Lianfa Y, Cheng G. Determination of stress-strain relationship of tubular materials with hydraulic bulge test. Thin Wall Struct 2008;46:147-54.

[8] Strano M, Altan T. An inverse energy approach to determine the flow stress of tubular materials for hydroforming applications. J Mater Process Techno 2004;146:92-6.

[9] Huang Y-M, Lin Y-K. Analysis and finite element simulation of the bulge hydroforming process. J Mater Process Technol 2002;125-126:821-5.

[10] Huang Y-M, Lin Y-K. Analysis of the tube bulge forming in an open-die considering anisotropic effects of the tubular material. Int J Mach Tools Manuf 2006;46:1921-8

[11] Velasco R, Boudeau N. Tube bulging test: theoretical analysis and numerical validation. J Mater Process Technol 2008;205:51-9.

[12] Koç M, Aue-u-lan Y, Altan T. On the characteristics of tubular materials for hydroforming-experimentation and analysis. Int J Mach Tools Manuf 2001;41:761-72.

[13] Bortot P, Ceretti E, Giardini C. The determination of flow stress of tubular material for hydroforming applications. J Mater Process Technol 2008;203: 381-8.

[14] Fuchizawa S, Narazaki M , Yuki H. Bulge test for determining stress-strain characteristics of thin tubes. In: Proceedings of the fourth international conference on technology of plasticity. Advanced technology of plasticity Vol. 1, 1993. p. 488-93.

[15] Kuwabara T, Yoshida K, Narihara K, Takahashi S. Anisotropic deformation of extruded aluminium alloy tube under axial forces and internal pressure. Int J Plasticity 2005;21:101-17.

[16] Gallée S Caractérisation expérimentale et simulation numérique des procédés d'emboutissage profond: application aux aciers inoxydables austénitiques. PhD thesis. France: Université de Bretagne-Sud, 2ème partie; 2005 p. 37-71.

[17] 〈www.matweb.com〉.

[18] Philibert J, Vignes A, Bréchet Y, Combrade P Métallurgie. Du minerai au matériau. Dunod, Collection Sciences Sup, 2ème édition.

[19] Boudeau N, Gelin JC. Prediction of the localized necking in 3D sheet meta forming processes from FE simulations. J Mater Process Technol 1994;45:229-35. 
[20] Boudeau N Prediction of local elasto-plastic instabilities. Application to deepdrawing. PhD thesis. France: University of Franche-comté; 1995. 172 p [in French].

[21] Banabic D. Anisotropy of sheet metal. In: Banabic D, editor. Formability of metallic materials. Plastic anisotropy, formability testing, forming limits. Berlin; Springer-Verlag; 2000. p. 119-68.

[22] Kyriakides S, Corona E. Plastic anisotropy in tubes. Mech Offshore Pipelines 2007; $1: 371-5$.
[23] Gutscher G, Wu H-C, Ngaile G, Altan T. Determination of flow stress for sheet forming using the viscous pressure bulge (VPB) test. J Mater Process Technol 2004; $149: 1-7$

[24] Ben Ouirane AH, Boudeau N, Velasco R, Michel G. Error evaluation on experimental stress-strain curve obtained from tube bulging test. Thin Wall Struct 2011;49:1217-24. 